\title{
Criação de uma escala de satisfação com a vida por meio da Teoria da Resposta ao Item
}

\author{
Development of a life satisfaction scale by Item Response Theory
}

Pablo Magno da Silveira', Adriano Ferreti Borgatto², Kelly Samara da Silva³, Elusa Santina Antunes de Oliveirat ${ }^{4}$ Mauro Virgílio Gomes de Barros', Markus Vinicius Nahas ${ }^{3}$

\section{RESUMO}

Objetivo: A satisfação com a vida é um julgamento cognitivo de alguns domínios específicos na vida e depende de uma comparação entre as circunstâncias de vida do indivíduo e um padrão por ele estabelecido. O objetivo deste estudo foi construir uma escala de satisfação com a vida por meio da Teoria da Resposta ao Item (TRI). Métodos: Para a criação da escala, foi utilizado o questionário "Estilo de Vida e Hábitos de Lazer de Trabalhadores da Indústria" e a Teoria da Resposta ao Item com base no modelo de resposta gradual proposto por Samejima. Participaram do estudo 47.477 trabalhadores brasileiros da indústria. Resultados: A escala contou com sete itens do bloco de perguntas "indicadores de saúde e comportamentos preventivos", com assuntos relacionados ao estado de saúde, qualidade de sono, nível de estresse, sentimento de tristeza ou depressão e percepção de vida no lar, no trabalho e no lazer. Conclusão: São poucos os estudos que tiveram seu foco direcionado à satisfação com a vida no Brasil, e aqueles que de alguma forma o abordaram incluíram somente uma questão específica sobre felicidade ou satisfação de vida, principalmente em estudos sobre o bem-estar subjetivo. Os resultados mostraram que a TRI foi eficiente para criar a escala, medindo principalmente aqueles indivíduos com valores intermediários de satisfação com a vida.

\section{ABSTRACT}

Objective: Life satisfaction is a cognitive judgment of some specific areas in life and depends on a comparison between the life circumstances of the individual and a standard established by it. The objective of this study was to construct a scale of satisfaction with life by Item Response Theory (IRT). Methods: For the creation of the scale we used the questionnaire "Life Style and Habits Leisure Industry Workers" and Item Response Theory based on the graduated response model proposed by Samejima. The study enrolled 47,477 Brazilian workers in the industry. Results: The scale had seven items the questions of the "health indicators and preventive behaviors" with issues related to health status, quality of sleep, stress level, feelings of sadness or depression and perception of life at home, at work and play. Conclusion: There are few studies that have their focus directed to life satisfaction in Brazil, and those who somehow discussed, only included a specific question about

1 Universidade Federal de Santa Catarina (UFSC), Núcleo de Pesquisa em Atividade Física e Saúde (NuPAF), Centro de Desportos. 2 UFSC, Departamento de Informática e Estatística, Centro Tecnológico.

3 UFSC, Centro de Desportos, Departamento de Educação Física. 


\section{Keywords}

Life satisfaction, workers industry, Item Response

Theory.

\section{INTRODUÇÃO}

A satisfação é um fenômeno complexo e de difícil mensuração, por se tratar de um estado subjetivo. Define-se com maior precisão a experiência de vida em relação às várias condições de vida do indivíduo'.

A satisfação com a vida é um julgamento cognitivo de alguns domínios específicos na vida como saúde, trabalho, condições de moradia, relações sociais, autonomia, ou seja, um processo de juízo e avaliação geral da própria vida de acordo com um critério próprio. O julgamento da satisfação depende de uma comparação entre as circunstâncias de vida do indivíduo e um padrão por ele estabelecido. Satisfação reflete, em parte, o bem-estar subjetivo individual, ou seja, o modo e os motivos que levam as pessoas a viverem suas experiências de vida de maneira positiva².

Ideologicamente, a satisfação com a vida tem suas origens no lluminismo do século XVIII, em que o propósito da vida humana é a própria vida. No século XIX, essa convicção é expressa no credo utilitário em que a melhor sociedade é aquela que fornece "a maior felicidade para o maior número de pessoas". No século XX, inspirados em tentativas planejadas de reforma social em grande escala, surge e se desenvolve o conceito de bem-estar ${ }^{3}$.

Atualmente, o tema bem-estar é recorrente na sociedade moderna e se apresenta como uma das principais preocupações das pessoas. Tal como os demais seres vivos, o ser humano busca a satisfação de suas necessidades, no entanto a consciência de sentir-se feliz é algo específico de nossa espécie ${ }^{4}$.

Tradicionalmente, aspectos subjetivos, tais como a satisfação, são mensurados por meio de uma pontuação simples, obtida por meio de perguntas relacionadas aos aspectos que se deseja medir. A pontuação obtida segue os princípios da Teoria Clássica dos Testes (TCT), a qual é fundamentada em uma pontuação baseada nas respostas do questionário (escore) e se torna uma medida, que pode ser usada para validar instrumentos ou fazer associações com fatores que estão relacionados a esse aspecto subjetivo. Como essa medida se baseia em uma pontuação do questionário, ela depende muito do instrumento escolhido, impossibilitando a comparação de resultados com instrumentos diferentes ${ }^{5-8}$.

Por outro lado, a Teoria da Resposta ao Item (TRI) aparece como uma das principais aplicações na área de psicometria, superando, na medição subjetiva, a teoria clássica. Algumas das limitações supridas pela TRI são a comparabilidade dos resultados usando diferentes instrumentos e a criação de uma escala em que os itens e os sujeitos são interpretados conjuntamente ${ }^{8,9}$. Além disso, a TRI permite mensurar um traço latente por meio de um conjunto de respostas a itens de um instrumento de avaliação ou de pesquisa. Portanto, o elemento principal para esse tipo de análise é o item, e não o instrumento, ou seja, a qualidade do instrumento é avaliada por cada item que o compõe ${ }^{10}$.

Esses modelos matemáticos são baseados na probabilidade de resposta do indivíduo, condicionada ao traço latente e às características dos instrumentos de medida ${ }^{11}$. O termo "traço latente" se refere a traços não observáveis ou diretamente mensuráveis, por exemplo, aptidões, habilidades, grau de satisfação e nível de estresse. Dessa forma, para medir o traço latente, é preciso o auxílio de um instrumento composto por itens, por exemplo, um questionário ${ }^{12}$. Neste trabalho, o traço latente que se pretende medir é a satisfação com a vida dos trabalhadores. Outra questão importante é que a TRI permite a criação de escalas de medida e, além disso, é possível posicionar na mesma escala os itens e os seus respondentes de acordo com o seu respectivo traço latente ${ }^{11}$.

Na literatura, a satisfação com a vida conta com diferentes métodos para avaliá-la ${ }^{13,14}$. Geralmente, a medida de satisfação com a vida mais utilizada consiste em um único item ${ }^{15}$. Neste estudo, é criada uma escala de medida com diferentes itens que retratam a satisfação em distintos ambientes frequentados ou inerentes ao cotidiano das pessoas. Nesse sentido, o presente estudo tem como objetivo apresentar, por meio da TRI, a criação de uma escala para avaliar a satisfação com a vida.

\section{MÉTODOS}

Este estudo é uma análise secundária de uma pesquisa nacional intitulada "Estilo de Vida e Hábitos de Lazer de Trabalhadores da Indústria", realizada pelo Serviço Social para Indústria (Sesi), em parceria com o Núcleo de Pesquisa em Atividade Física e Saúde, da Universidade Federal de Santa Catarina (NuPAF/UFSC), entre 2006 e 2008, com a participação de 24 das 27 unidades federativas do Brasil. Não participaram do inquérito em tempo hábil os estados do Rio de Janeiro, Piauí e Sergipe.

Cada Departamento Regional (DR) do Sesi, ou seja, cada uma das 24 unidades federativas do Brasil participantes da pesquisa possuía informações de cadastro e do número de trabalhadores de cada empresa industrial da unidade fede- 
rativa que representava. Essas informações foram utilizadas para o cálculo do tamanho da amostra e o planejamento amostral em cada DR.

Os tamanhos das amostras foram calculados separadamente para cada DR, usando a população-alvo e os seguintes parâmetros: a) prevalência estimada de $45 \%$ de inatividade física no lazer, obtida com base em um estudo anterior em que o objetivo primário do inquérito foi identificar a prevalência de inatividade física no lazer ${ }^{16}$; b) erro amostral de $3 \%$; c) intervalo de confiança de $95 \%$, d) efeito de delineamento de 1,5 e e) acréscimo de 20\% para eventuais casos de perdas e recusas. A amostra final foi composta por 52.774 trabalhadores recrutados em duas fases: 1 ) os trabalhadores foram estratificados de acordo com o tamanho da empresa empregadora, medido pelo número de trabalhadores, como se segue: pequeno (20-99), médio (100-499) e grande ( $\geq 500)$; 2) o número de trabalhadores foi estratificado de cada porte pelas Unidades Regionais, subdivisões de um Departamento Regional. O número de trabalhadores necessários de cada porte em cada subdivisão também seguiu a proporcionalidade encontrada na população de referência. Em seguida, as empresas foram selecionadas aleatoriamente. Em cada Unidade Regional foram sorteadas de $10 \%$ a $50 \%$ das empresas de cada porte, de acordo com o número de empresas existentes e o número de trabalhadores necessários para compor a amostra. Por fim, os trabalhadores foram sorteados de forma sistemática dentro das empresas selecionadas, por meio de listas fornecidas com os nomes de seus funcionários. Mais detalhes podem ser consultados em publicação anterior ${ }^{17}$. Quanto ao instrumento, foi utilizado um questionário previamente validado composto por 58 questões. Para esse estudo foi usado o bloco "Indicadores de Saúde e Comportamentos Preventivos", composto por 15 itens com informações relativas à percepção do estado de saúde atual, da qualidade do sono, da percepção dos níveis de estresse, bem-estar (no lar, no trabalho e no lazer), fumo, ingestão de bebidas alcoólicas, sentimento de tristeza e depressão, religião, proteção solar, hipertensão, colesterol elevado e diabetes.

Para a criação da escala de satisfação com a vida, foram atribuídos valores comparáveis aos itens e aos respondentes a fim de serem posicionados na mesma escala. De acordo com Araujo et al. ${ }^{10}$, uma das etapas mais importantes da TRI é a estimação dos parâmetros dos itens e dos traços latentes. A aplicação dos métodos de estimação para itens politômicos requer a utilização de ferramentas matemáticas complexas que necessitam de recursos computacionais e, para tal, neste trabalho os dados foram tratados por meio do software Multilog.

O processo de construção da escala se deu a partir da posse dos parâmetros dos itens, em um processo chamado de ancoragem. Ancorar um item em um nível da escala, segundo Andrade et al. ${ }^{11}$, envolve um processo estatístico que identifica o nível da escala em que o item apresenta maior discriminação. Já a definição da métrica, ou seja, da medida de posição e da medida de dispersão, é a base para a construção da escala. Após a especificação da métrica, a escala é interpretada no contexto do problema de pesquisa. Neste estudo, utilizou-se a escala $(0,1)$, ou seja, uma escala em que a média é 0 e o desvio-padrão é 1 .

Para posicionar os respondentes e os itens com respostas graduadas nesta escala, foi necessário utilizar um modelo probabilístico. Por causa da natureza dos itens, utilizou-se o modelo de resposta gradual, proposto por Samejima ${ }^{18}$. Nesse modelo, assume-se que as categorias de respostas de um item podem ser ordenadas entre si. Com isso, a probabilidade de um respondente $j$ escolher uma categoria $k(k=1,2,3$, 4) para um determinado item $i$ é dada por

$$
P_{i, k}(\theta)=\frac{1}{1+e^{-a_{i}\left(\theta_{j}-b_{i, k}\right)}}-\frac{1}{1+e^{-a_{i}\left(\theta_{j}-b_{i, k+1}\right)}}
$$

onde, por exemplo, um item que possui quatro categorias assume a restrição em que $b_{1}=0$, apresentando três valores do parâmetro de dificuldade na escala do estilo de vida $\left(b_{2}, b_{3}, b_{4}\right)$, além do parâmetro de discriminação do item $(a)$, o qual atribui o quanto cada item está associado à satisfação com a vida e os valores do traço latente $\left(\theta_{j}\right)$, ou seja, os valores da satisfação com a vida de cada respondente. Os parâmetros dos itens e o traço latente do respondente estabelecem a probabilidade de ele responder cada uma das categorias para um dado item. Essas probabilidades podem ser mais bem identificadas por meio das Curvas Características do Item (CCI). Cabe ressaltar que, neste modelo, deve-se ter uma ordenação entre os parâmetros de dificuldade das categorias de um dado item, ou seja, $b_{i, 1} \leq b_{i, 2} \leq b_{i, 3}$. O inquérito foi aprovado pelo Comitê de Ética em Pesquisa da Universidade Federal de Santa Catarina, Brasil (Pareceres no 306/2005 e no 009/2007). O Serviço Social da Indústria do Brasil (Sesi), entidade parceira na realização do inquérito, autorizou esta análise secundária de dados.

\section{RESULTADOS}

Para a avaliação dos itens e a criação da escala, estimaram-se os parâmetros dos itens. Antes de proceder com a análise dos dados, ressalta-se que o item 22 (Nos últimos 30 dias, você tomou 5 ou mais doses de bebida alcoólica em uma mesma ocasião?) do questionário foi eliminado, pois feriu um dos pressupostos da TRI, que é o da independência local, ou seja, a probabilidade de responder a um item é precisamente determinada pelo nível do traço latente do respondente, e não por suas respostas a outros itens do conjunto. Neste caso, a questão 22 é um complemento da questão 21 (Quantas doses de bebida alcoólica você toma em uma semana normal?). 
Após as estimativas dos parâmetros dos itens, mantiveram-se na análise itens com parâmetro de discriminação " $a$ " $>0,7$ (este valor é um indicativo que o item não esteja associado ao mesmo traço latente dos outros itens) ${ }^{19}$ e parâmetros de dificuldade $\left(b_{2^{\prime}}, b_{3^{\prime}} b_{4^{\prime}}, b_{5}\right)$ não muito além do intervalo -5 a 5 , bem como erro-padrão do parâmetro de um item não muito alto, em relação aos parâmetros dos demais itens de mesma magnitude (indicativo de inconsistência nas estimativas dos parâmetros). A tabela 1 apresenta os parâmetros dos 14 itens e seus respectivos erros-padrões.

Referente ao parâmetro de discriminação (a), conforme os valores apresentados na tabela 1 , sete itens $(16,20,21$, $23,24,25$ e 26 ) tiveram problemas, pois a $<0,7$ e, portanto, foram eliminados. A partir das considerações acima e da exclusão dos itens, foi necessário recalibrar os novos parâmetros dos itens. A tabela 2 apresenta os parâmetros dos itens e os respectivos erros-padrões, após a exclusão dos sete itens anteriormente detalhados.
Uma vez que todos os parâmetros dos itens e traços latentes dos respondentes estão numa mesma escala, ou seja, são comparáveis, pode-se construir a interpretação da escala. As escalas são definidas por níveis âncoras, que são os pontos da escala que serão interpretados. Esses pontos são caracterizados por um conjunto de categorias dos itens, de preferência itens âncora. Teoricamente, o critério adotado para o posicionamento do item na escala é feito usando dois níveis âncora consecutivos $Y$ e Z, com $Y<Z$. Uma categoria do item será âncora para o nível $Z$ se, e somente se, obedecer simultaneamente às condições abaixo:

$$
\begin{array}{ll}
\text { - } & P(U=1 \mid \theta=Z) \geq 0,65 \\
\text { - } & P(U=1 \mid \theta=Y)<0,50 \\
& P(U=1 \mid \theta=Z)-P(U=1 \mid q=Y) \geq 0,30
\end{array}
$$

Uma categoria do item é âncora se em um determinado nível da escala é respondido por pelo menos $65 \%$ dos respondentes com o mesmo nível do traço latente e por uma proporção de, no máximo, 50\% de respondentes com o nível

\begin{tabular}{|c|c|c|c|c|c|c|c|c|c|c|c|c|}
\hline ITEM & $\mathrm{a}$ & ep & $b_{2}$ & ep & $b_{3}$ & ep & $b_{4}$ & ep & $b_{5}$ & ep & $b_{6}$ & ep \\
\hline 12 & 1,09 & 0,01 & $-4,71$ & 0,08 & $-1,81$ & 0,03 & 1,44 & 0,02 & - & - & - & - \\
\hline 13 & 0,97 & 0,01 & $-4,35$ & 0.07 & $-1,60$ & 0,02 & 0,55 & 0,02 & - & - & - & - \\
\hline 14 & 1,11 & 0,01 & $-3,80$ & 0,05 & $-1,99$ & 0,03 & 0,56 & 0,01 & - & - & - & - \\
\hline 15 & 1,18 & 0,02 & $-4,57$ & 0,08 & -2.85 & 0.04 & 0,41 & 0,01 & - & - & - & - \\
\hline 16 & 0,44 & 0,01 & $-8,18$ & 0,26 & $-5,63$ & 0,18 & 0,57 & 0,03 & - & - & - & - \\
\hline 17 & 1,57 & 0,02 & $-4,81$ & 0,11 & $-3,75$ & 0,05 & $-1,61$ & 0,02 & 0,43 & 0,01 & - & - \\
\hline 18 & 1,45 & 0,02 & $-4,39$ & 0,08 & $-3,51$ & 0,05 & $-1,46$ & 0,02 & 0,96 & 0,01 & - & - \\
\hline 19 & 1,17 & 0.01 & $-4,21$ & 0,06 & $-2,61$ & 0,03 & $-0,73$ & 0,01 & 1,36 & 0,02 & - & - \\
\hline 20 & 0,45 & 0,01 & $-10,23$ & 0,35 & $-7,00$ & 0,23 & $-4,30$ & 0,14 & $-3,62$ & 0,12 & $-2,12$ & 0,07 \\
\hline 21 & 0,33 & 0,02 & $-10,60$ & 0,42 & $-7,48$ & 0,29 & $-0,92$ & 0,05 & - & - & - & - \\
\hline 23 & 0,32 & 0,01 & $-3,84$ & 0,15 & 0,12 & 0,04 & 3,02 & 0,11 & - & - & - & - \\
\hline 24 & 0,68 & 0,05 & $-6,17$ & 0,42 & - & - & - & - & - & - & - & - \\
\hline 25 & 0,43 & 0,02 & $-5,49$ & 0,29 & - & - & - & - & - & - & - & - \\
\hline 26 & 0,45 & 0,02 & $-5,10$ & 0,25 & - & - & - & - & - & - & - & - \\
\hline
\end{tabular}

Tabela 1. Estimativas dos parâmetros dos itens para cada categoria de resposta e seus respectivos erros-padrões (ep) da primeira rodagem

* ep: erro-padrão.

\begin{tabular}{|c|c|c|c|c|c|c|c|c|c|c|}
\hline ITEM & A & ep* & $b_{2}$ & ep & $b_{3}$ & ep & $b_{4}$ & ep & $\mathbf{b}_{5}$ & ep \\
\hline 12 & 1,11 & 0.01 & $-4,68$ & 0.07 & $-1,80$ & 0.02 & 1,43 & 0,02 & - & - \\
\hline 13 & 0,98 & 0,01 & $-4,32$ & 0,06 & $-1,59$ & 0,02 & 0,55 & 0,01 & - & - \\
\hline 14 & 1,14 & 0,01 & $-3,74$ & 0,05 & $-1,96$ & 0,02 & 0,55 & 0,01 & - & - \\
\hline 15 & 1,22 & 0,02 & $-4,49$ & 0,07 & $-2,80$ & 0,03 & 0,40 & 0,01 & - & - \\
\hline 17 & 1,48 & 0,02 & $-5,03$ & 0,11 & $-3,91$ & 0,05 & $-1,67$ & 0,02 & 0,44 & 0,01 \\
\hline 18 & 1,46 & 0,02 & $-4,40$ & 0,07 & $-3,52$ & 0,04 & $-1,46$ & 0,02 & 0,97 & 0,01 \\
\hline 19 & 1,19 & 0,01 & $-4,17$ & 0,06 & $-2,59$ & 0,03 & $-0,73$ & 0,01 & 1,35 & 0,02 \\
\hline
\end{tabular}

Tabela 2. Estimativas dos parâmetros dos itens para cada categoria de resposta e seus respectivos erros-padrões (ep) da segunda rodagem

* ep: erro-padrão 
do traço latente imediatamente anterior. Além disso, a diferença entre a proporção de respondentes nesses níveis da satisfação com a vida que marcaram esse item deve ser de, pelo menos, 30\% ${ }^{11}$. Neste estudo, a fim de enriquecer a interpretação da escala, pois o estudo se baseia em sete itens, decidiu-se posicionar todas as categorias dos itens mesmo que não atendendo a todos os critérios de âncora.

A figura 1 apresenta a Cll, após terem sido realizados todos os ajustes necessários.

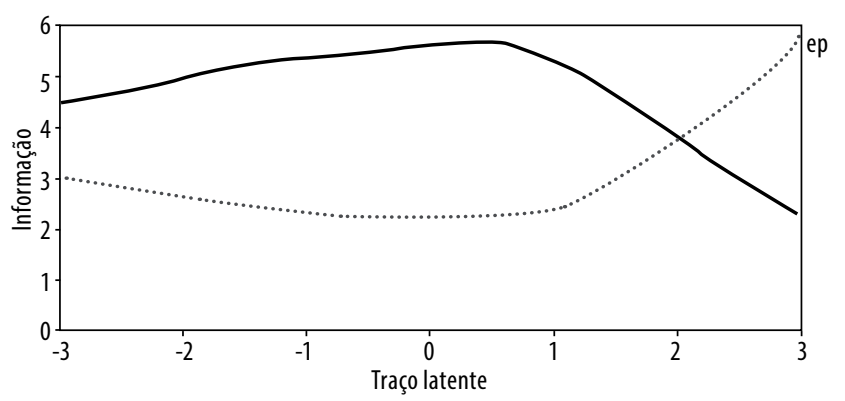

ep: erro-padrão.

Figura 1. Curva de informação do instrumento e erro-padrão.

A curva de informação do instrumento demonstra que a maior quantidade de informação está no intervalo de -3 a 2, o que significa dizer que ele é mais adequado para medir a satisfação com a vida de indivíduos que possuem seus níveis entre -3 e 2. Quanto menor o erro-padrão (linha pontilhada), maior será a informação contida nesse determinado intervalo.

Após essa análise, o traço latente de cada indivíduo foi estimado. $\mathrm{O}$ traço latente assume uma posição na escala de medida e representa a satisfação com a vida dos trabalhadores. Consequentemente, quanto maior o valor do traço latente, melhor é a satisfação com a vida.

Observa-se, na figura 2, que as categorias dos itens que apresentam nível muito bom de satisfação com a vida, representado pelos parâmetros $b_{4}$ (itens com quatro categorias de resposta) e $b_{5}$ (itens com cinco categorias de resposta), estão posicionadas a partir do intervalo 0-1. Nota-se também que $11,7 \%$ dos trabalhadores apresentam as categorias de respostas mais positivas, ou seja, são trabalhadores com a satisfação com a vida no intervalo 1-2.

De acordo com os critérios de interpretação estabelecidos, pode-se dizer que, para os trabalhadores brasileiros da indústria, os itens 12 e 19 tiveram os parâmetros associados à satisfação com a vida "excelente", já que seus parâmetros $b_{4}$ e $b_{5}$ estão posicionados acima dos demais itens. Isso significa que somente aqueles trabalhadores que apresentaram valores mais elevados do traço latente (satisfação com a vida) possuem alta probabilidade de classificar como "excelente" seu estado de saúde e de se sentir "muito bem" em relação ao seu lazer. Consequentemente, são os itens que esses trabalhadores têm mais dificuldade de responder de forma

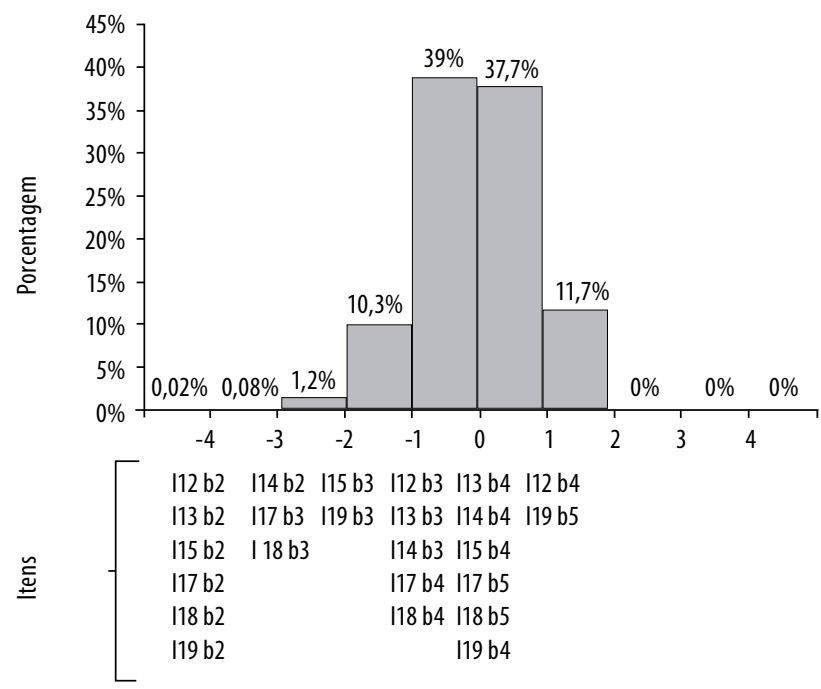

Figura 2. Posicionamento das categorias dos itens e a distribuição dos valores da satisfação com a vida dos trabalhadores brasileiros da indústria $(0 ; 1)$.

mais positiva. Por outro lado, os itens que os trabalhadores têm mais facilidade de responder de forma mais positiva são os itens 17 e 18, que tratam da relação de sentimento de vida no lar e no trabalho, pois os parâmetros $b_{4^{\prime}}$ ou seja, as categorias de respostas mais positivas do item, estão posicionados antes dos demais parâmetros dos outros itens.

Para a construção da escala, definiram-se quatro níveis de satisfação com a vida, a saber: ruim, regular, bom e excelente. Pode-se afirmar ainda que todo respondente com excelente satisfação com a vida apresenta grande probabilidade de que todos os sete itens sempre façam parte da sua vida. A descrição de cada um desses níveis está apresentada no quadro 1.

Quadro 1. Interpretação da escala de satisfação com a vida

\begin{tabular}{|c|c|c|c|}
\hline Nível & Intervalo & $\%$ & Descrição dos níveis \\
\hline Ruim & $<-3$ & $0,1 \%$ & $\begin{array}{l}\text { Neste nível o respondente classifica como regular } \\
\text { seu estado de saúde, às vezes dorme bem, quase } \\
\text { sempre está triste ou deprimido e se sente mal em } \\
\text { relação a sua vida no lar, no trabalho e no lazer. }\end{array}$ \\
\hline Regular & {$[-3 ;-1)$} & $11,5 \%$ & $\begin{array}{l}\text { Neste nível, o respondente classifica como regular } \\
\text { seu estado de saúde, às vezes dorme bem, quase } \\
\text { sempre está estressado, às vezes está triste ou } \\
\text { deprimido e se sente mais ou menos em relação a } \\
\text { sua vida no lar, no trabalho e no lazer. }\end{array}$ \\
\hline Bom & {$[-1 ; 1)$} & $76,7 \%$ & $\begin{array}{l}\text { Neste nível, o respondente classifica como bom seu } \\
\text { estado de saúde, sempre dorme bem, raramente } \\
\text { está estressado, nunca/raramente está triste ou } \\
\text { deprimido, se sente muito bem em relação sua vida } \\
\text { no lar e no trabalho e se sente bem em relação a } \\
\text { sua vida no lazer. }\end{array}$ \\
\hline Excelente & $\geq 1$ & $11,7 \%$ & $\begin{array}{l}\text { Neste nível, o respondente classifica como } \\
\text { excelente seu estado de saúde, sempre dorme } \\
\text { bem, raramente está estressado, nunca/raramente } \\
\text { está triste ou deprimido e se sente muito bem em } \\
\text { relação a sua vida no lar, no trabalho e no lazer. }\end{array}$ \\
\hline
\end{tabular}




\section{DISCUSSÃO}

O objetivo principal deste estudo foi criar uma escala de satisfação com a vida com o auxílio de uma amostra representativa dos trabalhadores brasileiros da indústria. A medida criada neste estudo une variáveis importantes para descrever satisfação com a vida. Estão evidenciadas na literatura outras formas de medir satisfação com a vida comum único item ${ }^{20,21}$ e com mais de um item ${ }^{13}$.

São poucas as informações sobre a satisfação com a vida no Brasil, principalmente em uma população de industriários. De fato, são poucos os estudos que tiveram seu foco direcionado ao tema e aqueles que de alguma forma o abordaram incluíram somente uma questão específica sobre felicidade ou satisfação de vida, principalmente em estudos sobre o bem-estar subjetivo.

Foi observado na literatura que a denominação "felicidade" ou "satisfação com a vida" é pouco utilizada, sendo substituída geralmente pelo termo "bem-estar subjetivo"22,23. Isso se deve à dificuldade em abordar diretamente a felicidade ou a satisfação com a vida, de modo que a mensuração do bem-estar subjetivo se torna mais habitual ${ }^{24}$. A literatura ainda considera confundíveis os termos "felicidade", "satisfação com a vida", "qualidade de vida" e "bem-estar", pois estes denotam o mesmo significado e muitas vezes são utilizados como sinônimos ${ }^{25}$. Angeles ${ }^{26}$ ainda destaca que as pesquisas sobre satisfação com a vida e felicidade mostram alta correlação.

A TRI vem demonstrando em diversos estudos ser eficiente na construção de escalas de medida ${ }^{19-28}$. Não se contatou na literatura o uso da TRI para a criação de uma escala de satisfação com a vida, como proposto neste trabalho.

Neste estudo, verificou-se que $11,6 \%$ dos trabalhadores possuem índices ruim-regular de satisfação com a vida, com valores bem abaixo da média e 11,7\% possuem índice excelente de satisfação com a vida. A grande maioria, 76,7\%, possui boa satisfação com a vida, com valores intermediários, bem próximos à média. Resultado diferente foi encontrado em uma grande amostra representativa de adultos dos Estados Unidos. Ao avaliarem a satisfação na vida por meio de uma medida de único item, revelaram que $94,4 \%$ dos americanos estavam satisfeitos ${ }^{29}$. Contudo, a prevalência de boa satisfação com a vida encontrada neste estudo foi próxima à de inquéritos sobre bem-estar conduzidos na Austrália $(70,4 \%)$ e na Tailândia $(70 \%)^{30,31}$.

Os resultados ainda se mostraram positivos em três aspectos: a) abordou os recursos da TRI para criação de um índice; b) evidenciou o estudo da satisfação com a vida; e c) para isso, utilizou um banco de dados com uma amostra representativa dos trabalhadores da indústria, com participação de 24 das 27 unidades federativas do Brasil.

\section{CONCLUSÃO}

Por meio da curva de informação do instrumento, pode-se concluir que a maior quantidade de informação está na parte central da escala (nível bom), revelando a potencialidade para medir a satisfação com a vida de indivíduos com traço latente entre o intervalo -1 e 1. Neste estudo a maior parte dos trabalhadores $(76,7 \%)$ ficou posicionada no nível bom, indicando uma tendência de boas percepções em relação aos aspectos de vida abordados nos sete itens da escala.

\section{CONTRIBUIÇÕES INDIVIDUAIS}

Pablo Magno da Silveira - Participou da concepção do estudo, revisão bibliográfica, interpretação dos dados, redação, revisão crítica e aprovação final do artigo.

Adriano Ferreti Borgatto - Participou da concepção do estudo, das análises e interpretação dos dados, redação, revisão crítica e aprovação final do artigo.

Kelly Samara da Silva - Participou da concepção do estudo, redação, revisão crítica e aprovação final do artigo.

Elusa Santina Antunes de Oliveira, Mauro Virgílio Gomes de Barros e Markus Vinicius Nahas - Elaboraram e coordenaram o projeto, gerenciaram a coleta de dados e participaram da concepção do manuscrito, revisão crítica e aprovação final do artigo.

\section{AGRADECIMENTO}

Ao Serviço Social da Indústria (Sesi).

\section{CONFLITOS DE INTERESSE}

Os autores deixam claro não haver conflitos de interesse.

\section{REFERÊNCIAS}

1. Spirduso WW. Physical dimensions of aging. Champaign: Human Kinetics; 1995.

2. Albuquerque AS, Troccoli BT. Desenvolvimento de uma escala de bem-estar subjetivo. Psicol Teor Pesq. 2004;20(2):153-64.

3. Veenhoven R. El estudio de la satisfacción con la vida. Interv Psicosoc. 1994;3:87-116.

4. Seligman ME, Csikszentmihalyi M. Positive psychology. An introduction. Am Psychol. 2000;55(1):5-14.

5. Hambleton RK. Emergence of item response modeling in instrument development and data analysis. Med Care. 2000;38(9 Suppl):1160-5

6. Nunnally JC. Psychometric theory. New York: McGraw Hill; 1967.

7. Gulliksen H. Theory of mental tests. New York: Wiley; 1950. 484p.

8. Nguyen TH, Han HR, Kim MT, Chan KS. An introduction to Item Response Theory for patient-reported outcome measurement. Patient. 2014;7(1):23-35. 
9. Embretson SE, Reise SP. Item Response Theory for psychologists. Mahwah, New Jersey, London: Lawrence Erlbaum Associates; 2000.

10. Araujo EAC, Andrade DF, Bortolotti SLV. Teoria da Resposta ao Item. Rev Esc Enferm USP. 2009;43(Esp):1000-8.

11. Andrade DF, Tavares HR, Valle RC. Teoria da Resposta ao Item: conceitos e aplicações. ABE - Associação Brasileira de Estatística; 2000.

12. Pasquali L, Primi R. Fundamentos da teoria da resposta ao item: TRI. Aval Psicol. 2003;2(2):99-110.

13. Diener E, Emmons RA, Larsen RJ, Griffin S. The Satisfaction With Life Scale. J Pers Assess. 1985;49(1):71-5.

14. Lawrence RH, Liang J. Structural integration of the Affect Balance Scale and the Life Satisfaction Index A: race, sex, and age differences. Psychol Aging. 1988;3(4):375-84.

15. Krause NM, Jay GM. What do global self-rated health items measure? Med Care. 1994;32(9):930-42.

16. Barros MVG, Nahas MV. Comportamentos de risco, autoavaliaçäo do nível de saúde e percepçäo de estresse entre trabalhadores da indústria. Rev Saúde Pública. 2001;35(6):554-63.

17. Nahas MV, Barros MV, Oliveira ES, Aguiar FS. Estilo de vida e hábitos de lazer dos trabalhadores das indústrias brasileiras: relatório geral. Brasília; 2009.

18. Samejima FA. Estimation of latent ability using a response pattern of graded scores. Psychometric Monograph; 1969.

19. Tezza R. Proposta de um construto para medir usabilidade em sites de e-commerce utilizando a Teoria da Resposta ao Item [dissertação]. Florianópolis, SC: Universidade Federal de Santa Catarina; 2009.
20. Cantril H. The pattern of human concerns. N. Brunswick: Rutgers University Press; 1965.

21. Campbell A. Subjective measures of well-being. Am Psychol. 1976;31(2):117-24.

22. Cloninger CR. Feeling good: the science of well-being. New York: Oxford University Press; 2004.

23. Diener E, Biswas-Diener R. Will money increase subjective well-being? Social Indic Res. 2002;57(2):119-69.

24. Ferraz RB, Tavares H, Zilberman ML. Felicidade: uma revisão. Arch Clin Psychiatry 2007;34:234-42

25. Veenhoven R. The four qualities of life: ordering concepts and measures of the good life. J Happiness Stud. 2000;1:1-39.

26. Angeles L. Children and Life Satisfaction. J Happiness Stud. 2010;11(4):523-38.

27. Gomes DE. Criação de uma escala para avaliar a percepção de funcionários de hospitais universitários no Brasil quanto ao ambiente organizacional [dissertação]. Florianópolis: UFSC; 2014.

28. Testa WL. Criação de uma escala da qualidade de vida para profissionais de educcação física por meio da Teoria da Resposta ao Item [dissertação]. Florianópolis: UFSC; 2014.

29. Strine TW, Chapman DP, Balluz LS, Moriarty DG, Mokdad AH. The associations between life satisfaction and health-related quality of life, chronic illness, and health behaviors among U.S. community-dwelling adults. J Community Health. 2008;33(1):40-50.

30. Dear K, Henderson S, Korten A. Well-being in Australia--findings from the National Survey of Mental Health and Well-being. Soc Psychiatry Psychiatr Epidemiol. 2002;37(11):503-9.

31. Yiengprugsawan V, Seubsman S, Khamman S, Lim LLY, Sleigh AC; Thai Cohort Stud Team. Personal Wellbeing Index in a National Cohort of 87,134 Thai adults. Soc Indic Res. 2010;98(2):201-15. 